\title{
Supporting Information \\ A Novel Stereocomplex Poly(lactic acid) with Shish-Kebab \\ Crystals and Bionic Surface Structures as Bioimplant Materials \\ for Tissue Engineering Applications
}

\author{
Jiafeng $\mathrm{Li}^{\text {a }}$, Wuyou Ye a ${ }^{\text {a }}$ Zhongyong Fan ${ }^{\mathrm{a}^{*}}, \mathrm{Lu} \mathrm{Cao}^{\mathrm{b}^{*}}$ \\ a Department of Materials Science, Shanghai Stomatological Hospital, Fudan University, Shanghai, P. R. \\ China \\ b Department of Orthopedic Surgery, Zhongshan Hospital, Fudan University, Shanghai, P. R. China \\ * Corresponding author: Zhongyong Fan, Department of Materials Science, Fudan University, Shanghai, P. \\ R. China, Tel: +86-21-65642395, Email: zyfan@fudan.edu.cn
}

Lu Cao, Department of Orthopedic Surgery, Zhongshan Hospital, Fudan University, Shanghai, P. R. China, Tel:

+86-21-64041990-2908, Email: caolu922@126.com 


\section{Preparation of Long Chain Branching PLA}

In vacuum at $70^{\circ} \mathrm{C}$ for $5 \mathrm{~h}$ dealt with PLA pellets and PDMA prepared the premixes for long chain branching PLA in a single screw extruder with a rotating speed of 90 $\mathrm{rpm}$ at $180^{\circ} \mathrm{C}$. And then the premixes mixed with PFE processed by in-situ reactive extrusion for 15 minutes to prepare b-PLAs. As shown in Figure S1, the topology structures of b-PLAs samples are composed of linear, symmetric-star, asymmetric-star, H-type, pom-pom, comb, and tree-like chains.
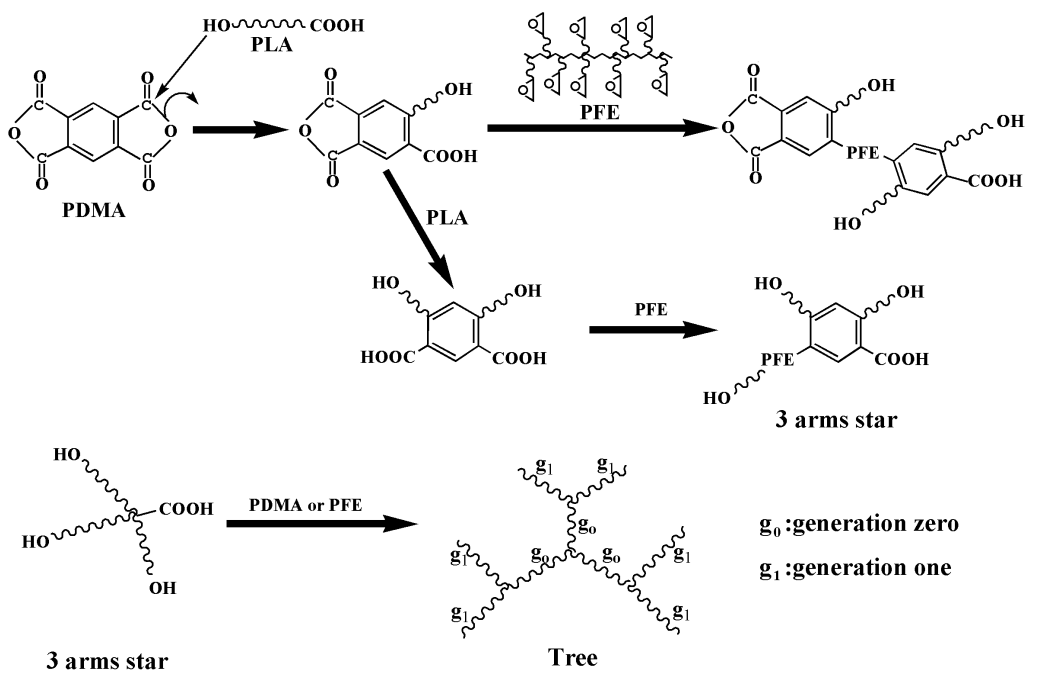

Figure S1 The reactive process and chains configuration of the b-PLAs 